\title{
L'anxiété nuisible chez les apprenants du FLE. Le cas des étudiants de la philologie romane en cycle de licence
}

\section{The debilitating language anxiety of learners of French as a foreign language. The case of students of Romance philology in first cycle studies}

\begin{abstract}
Language anxiety is the feeling of tension and apprehension associated with second / foreign language learning. It manifests itself in two types of emotional reactions: positive (positive anxiety) and negative (debilitating anxiety). The focus of this article is to explore the debilitating language anxiety of students learning French as a foreign language and to find out its level in the first, second and third years of undergraduate studies in Romance philology. The main research problem concerns the need to develop their ability to manage debilitating language anxiety. For this purpose, the findings of a FLCAS questionnaire are presented.
\end{abstract}

KEYWORD: debilitating language anxiety, teaching French as a foreign language, student.

MOTS-CLÉS: anxiété langagière débilitante, français langue étrangère, étudiant.

L'anxiété langagière peut soit engendrer une augmentation de l'effort des apprenants qui «semblent se servir de leur anxiété pour intensifier leurs efforts en vue de la réussite » (Brever $2010: 77$ ) soit les perturber voire les «freiner ». Selon les chercheurs, elle se manifeste par deux types de réactions émotionnelles : positive (l'anxiété positive) et négative (l'anxiété nuisible) (Łuszczyńska 2017). Dans les études sur les rapports entre l'anxiété langagière et la performance de l'apprenant, la confusion des types d'anxiété et le recours aux différents instruments de mesure non standardisés conduisent selon T. Scovel (1978) à des résultats de recherches contradictoires. Certaines recherches montrent que l'anxiété 
langagière gêne la performance de l'apprenant, d'autres prouvent au contraire qu' elle a des effets positifs sur l'apprentissage (MacIntyre 1995). D'autres encore soulignent que l'anxiété langagière n'est pas le facteur unique de l'échec scolaire (Łuszczyńska 2017).

Cet article s'intéresse à l'anxiété dite nuisible ou débilitante, chez les étudiants en philologie romane. Pendant divers cours de français / en français en cycle de licence, on a repéré le clivage entre les tensions et les peurs observées chez certains étudiants et les opportunités du développement langagier / interpersonnel offertes par les études de licence à l'université Adam Mickiewicz, lesquelles se déroulent dans une ambiance de sécurité voire de bienveillance. Cette observation a conduit à constater la nécessité de mesurer le niveau d'anxiété langagière nuisible chez lesdits étudiants en cycle de licence afin de préciser la taille de ce phénomène et de voir s'il y a un besoin de remédier à ses conséquences néfastes pour l'apprentissage du FLE dans le contexte universitaire. On est parti de la prémisse que l'anxiété langagière nuisible constitue un défi pédagogique potentiel pour l'enseignement du FLE en cycle de licence. La validité de cette prémisse est discutée dans le présent article. On commence par les caractéristiques de l'anxiété langagière nuisible, on passe ensuite à l'exposition et à la dicussion des résultats d'une enquête mesurant le niveau d'anxiété langagière nuisible chez les étudiants du cycle de licence afin d'aboutir aux conclusions sur les démarches pédagogiques à entreprendre.

\section{L'ANXIÉTÉ LANGAGIÈRE EN TANT QU'ÉLÉMENT DÉBILITANT L'APPRENTISSAGE}

Le concept d'anxiété relative à la situation d'apprentissage des langues étrangères (LE) / secondes (LS), a été proposé dans les années quatre-vingts du siècle dernier par E. K. Horwitz, M. B. Horwitz et J. A. Cope (1986). Les chercheurs précisaient que l'anxiété langagière était responsable des réactions émotionnelles négatives affectant la performance de l'apprenant en classe de LE / LS. D'autres recherches confirment la spécificité de l'anxiété langagière (Young 1992 ; Zhang 2001) et « démontrent que des apprenants ne souffrant pas d'anxiété particulière devant d'autres types d'apprentissages (celui des mathématiques, par exemple) en éprouvaient quand il s'agissait d'acquérir une L2 » (Jebali 2018:3). Selon A. Jebali (2018:3) cette spécificité s'explique "par le fait que l'apprenant d'une L2 se sent démuni lorsqu'il est privé du confort que lui confère sa langue première (L1) pour s'exprimer ou entreprendre les tâches de communication dans une L2 ». 
Soulignant l'impact du contexte institutionnel de l'apprentissage des langues sur l'émergence de sentiments d'insuffisance et de la peur de l'échec, E. K. Horwitz, M. B. Horwitz et J. A. Cope définissent l'anxiété langagière comme « un tout composé d'auto-perceptions, un concept distinct et complexe composé de perceptions de soi, de croyances, de sentiments et de comportements liés à l'apprentissage des langues en classe, découlant du caractère unique de cet apprentissage $»^{1}$ (Horwitz et al. $1986: 128$ ). Pour évaluer son influence sur l'apprentissage de LE / LS E. K. Horwitz, M. B. Horwitz et J. A. Cope ont élaboré un instrument de mesure : "Foreign Language Classroom Anxiety Scale » (FLCAS) (Horwitz et al. 1986). Le FLCAS évalue les effets cognitifs, personnels et sociaux de l'anxiété langagière nuisible en classe de langue. Il mesure trois types d'anxiété relative à la situation d'apprentissage des LE / LS : la peur de communication, l'anxiété face aux tests (dite " la peur d'échouer à l'examen ») et la peur de l'évaluation négative. Passons à leurs brèves caractéristiques.

1. La peur de communication peut se relever dans toutes les situations de communication. Dans l'apprentissage des LE / LS elle se manifeste quand l'apprenant se sent incapable de comprendre ou de s'exprimer en classe de langue devant un enseignant et / ou devant ses pairs (Horwitz et al. 1986 ; Young 1986 ; Daly 1991 ; MacIntyre \& Gardner 1994b : 284).

2. L'anxiété face aux tests apparaît quand les apprenants se focalisent sur leurs lacunes, leurs expériences négatives, leurs échecs vécus, ce qui détourne leur attention de la tâche assignée (Wine 1971 ; Sarason 1987). Elle n'est pas spécifique à l'apprentissage des LE / LS mais elle est susceptible d'être provoquée par les examens de langues étrangères, en particulier les examens oraux (MacIntyre \& Gardner 1991 : 105).

3. La peur de l'évaluation négative est fondée entre autres sur la conviction de l'individu que les autres l'évalueront négativement. L'individu craint donc les évaluations formulées par les autres et il esquive les situations d'évaluation (Horwitz et al. 1986 : 128). En classe de langue, il s'agit de la peur de commettre des erreurs, d'être corrigé par l'enseignant devant toute la classe. En général, le sujet apprenant craignant une évaluation négative, pense être plus faible en langue que ses pairs. Il préfère donc ne rien dire et évite la participation aux interactions qui se déroulent en classe.

L'anxiété langagière est en corrélation avec de nombreux facteurs qui se rapportent à l'enseignant, au sujet apprenant, à la matière enseignée et à l'environnement socio-culturel. S. Kalińska-Łuszczyńska (2016 : 75) les regroupent en trois catégories :

\footnotetext{
${ }^{1}$ Traduction propre.
} 
- les facteurs personnels incluant le sexe, la peur de l'évaluation négative, l'autoévaluation de la compétence en production orale, les traits de personnalité (tels que le perfectionnisme et l'introversion, la peur de perdre la face),

- les facteurs éducationnels comprenant les styles d'apprentissage et d'enseignement, les attitudes des enseignants, la difficulté des tâches et des activités, les façons de gérer l'anxiété, les types d'activités réalisées en classe, les expériences d'apprentissages négatives,

- les facteurs sociaux englobant le soutien social, les difficultés économiques et les événements critiques.

L'anxiété langagière est donc un trait de la personnalité ou une disposition comportementaliste de l'individu de percevoir certaines situations d'apprentissage comme une menace et d'y réagir par une tension et une appréhension (MacIntyre \& Gardner 1994a) donc d'entrer dans un état d'anxiété (Kolber 2008 : 123). Elle se manifeste lorsque l'activité pose des exigences qui risquent de dépasser les ressources et les capacités (Williams 1991) du sujet apprenant et / ou le degré de sa maîtrise de la langue cible (Gardner \& MacIntyre 1993). Elle est " comme une émotion psychologique et / ou une réaction physiologique qui gêne la concentration, l'attention et l'effort de l'apprenant en l'empêchant de maîtriser la tâche assignée» (Kamińska-Łuszczyńska 2015 : 37) dans différents contextes d'apprentissage : la lecture, l'écriture, la compréhension orale, l'expression orale (Gardner \& MacIntyre 1993 : 5). De nombreux chercheurs admettent pourtant que les situations provoquant le plus de tension et d'appréhension, sont celles de l'expression orale en classe de langue et celles de la compréhension orale (Kolber 2008 ; Horwitz et al. 1986 ; Hilleson 1996 ; MacIntyre \& Gardner 1993 ; Matsuda \& Gobel 2004 ; Young 1992 ; Phillips 1991 ; Porcher 1995).

Plus le degré d'anxiété est élevé, plus ses effets sur la performance langagière de l'apprenant sont négatifs. Le haut degré d'anxiété langagière peut affecter les performances cognitives et linguistiques du sujet apprenant. Par conséquent ses jugements, sa mémorisation, son analyse des situations, ses prises de décisions sont nettement moins bons et il commet plus d'erreurs : " le non-emploi de certaines formes pourtant bien assimilées, l'absence de cohésion du discours et une certaine déviation de la norme déjà apprise (grammaticale, sociolinguistique, pragmatique) » (Jebali $2018: 10)$.

En bref, l'anxiété langagière est un phénomène socio-psycho-biologique, complexe et multidimentionnel (Kamińska-Łuszczyńka 2015), relatif à la situation d'apprentissage de LE / LS. L'anxiété nuisible gêne les efforts, la concentration et l'attention de l'apprenant affectant ainsi sa performance. Au lieu de prendre pleinement plaisir à pratiquer le français, il alloue une partie de ses ressources pour contrôler ses peurs de ne pas comprendre ou de ne pas être compris, de ne pas donner la bonne réponse et enfin d'être sûrement évalué négativement. 


\section{GÉNÈSE DU PROBLÈME ET QUESTIONS DE RECHERCHE}

Durant le cycle de licence, les étudiants en philologie romane de l'Université Adam Mickiewicz suivent les cours de français dirigé et les cours en français (par exemple de littérature ou d'histoire). Lors de l'année universitaire 2018/2019 leur nombre total d'heures s'élevait à la fin du cycle de licence, à 1170 heures pour les étudiants débutants / intermédiaires et 1050 heures pour les étudiants des groupes avancés. Quant aux cours de français dirigé, leur nombre total d'heures s'élevait à 780 heures pour les débutants / intermédiaires et 660 heures pour les avancés. Le programme des cours de français dirigé est conçu suivant les principes $\mathrm{du}$ "Cadre européen commun de références pour les langues : apprendre, enseigner, évaluer. Volume complémentaire avec de nouveaux descripteurs » (2018) et réalisé dans le cadre de la méthodologie de l'approche communicative / actionnelle. Les cours de français dirigé visent le développement des compétences langagières, interpersonnelles et créatives des étudiants. Ils leur offrent une bonne opportunité pour les exercer et les approfondir. Ces étudiants y répètent à maintes reprises les mêmes types d'activités, revivent les mêmes types de situations en classe de langue (interrogatoires, prise de parole, discussion, test d'écoute, etc.) et se familiarisent aux rituels universitaires en confrontant leurs attentes à la réalité académique. C'est ainsi qu'ils construisent leur expérience d'études.

Il est à souligner que les procédures et les techniques utilisées en cours de français dirigé sont en principe focalisées sur l'atténuation de l'anxiété langagière des étudiants, à savoir : le recours aux jeux, le travail en binôme, les examens conçus à partir du contenu des cours, les critères et principes d'évaluation uniformes pour le module des cours de français dirigé, l'information sur les critères et principes d'évaluation distribuée au début de chaque semestre pendant tous les cours de français dirigé. De plus, considéré comme partenaire du processus d'enseignement / apprentissage, l'étudiant est encouragé à donner un retour d'expérience sur les cours suivis.

Il semble que ces conditions d'études devraient plutôt réconforter les étudiants, les encourager à participer activement aux cours, pour en tirer le meilleur parti sans se soucier trop des fautes ou des opinions de leurs pairs. Pourtant, on les a assez souvent entendu avouer les uns aux autres leurs inquiétudes et leurs peurs devant les situations d'apprentissage institutionnel. On a pu également repérer quelques symptômes physiologiques ou comportementaux de l'anxiété : les mains moites, une forte transpiration et la négligence des devoirs. C'est pourquoi on était tenté de savoir dans quelle mesure les réactions émotionnelles négatives perturbaient la performance des étudiants représentant un niveau de connaissance langagière et une expérience d'études différentes. On s'est donc posé les questions qui suivent : 
1. Quel est le degré d'anxiété langagière générale éprouvé par les étudiants de la $1^{\text {ère }}$ de la $2^{\text {ème }}$ et de la $3^{\text {ème }}$ année du cycle de licence?

2. Quel est le degré d'anxiété langagière relative à la peur de communication, la peur face aux tests et la peur de l'évaluation négative dans les groupes interrogés?

\section{PUBLIC CIBLE ET MÉTHODOLOGIE}

Pour répondre aux questions ci-dessus, on a proposé aux étudiants du cycle de licence de remplir l'adaptation française du questionnaire «FLCAS » (Horwitz et al.) élaborée par L. Jarie, C. S. Bordás, A. M. Orozco et P. U. Supervía (2019) (voir Annexe 1). L'enquête a été effectuée sur l'année universitaire 2018/2019 auprès des étudiants de la philologie romane de l'Université Adam Mickiewicz se trouvant au début, au milieu et à la fin de leur parcours universitaire en cycle de licence. On a reçu 93 réponses dont :

- 44 de la part des étudiants de la 1 ère année (18 du groupe débutant, 12 du groupe intermédiaire et 14 du groupe avancé). Ces étudiants se situaient au degré $\mathrm{A} 2+$ de français à la fin de leur $2^{\text {ème }}$ semestre d'études,

- 26 de la part des étudiants de la $2^{\text {ème }}$ année (12 du groupe intermédiaire et 14 du groupe avancé). À la fin de leur $4^{\text {ème }}$ semestre d'études, ils étaient au niveau $\mathrm{B} 1+$ de français,

- 26 de la part des étudiants de la $3^{\text {ème }}$ année achevant leur $6^{\text {ème }}$ semestre d'études et ayant le degré B2+ de français.

L'adaptation du « FLCAS » a été complétée par une consigne pour les étudiants et les 33 items du questionnaire ont été réparties selon les trois composantes de l'anxiété langagière distinguées par E. K. Horwitz, M. B. Horwitz et J. A. Cope (1986) (voir Tableau 1.). Il convient de préciser que le coefficient de fiabilité de la version française du FLCAS $(0,77)$ indique sa bonne cohérence interne. Par ailleurs, la fiabilité et la validité du FLCAS ont été testées par de nombreux chercheurs et E. K. Horwitz et ses collaborateurs (1986) ont effectué leur test avec 300 étudiants de langue étrangère.

Tableau 1. Analyse des items du questionnaire

\begin{tabular}{|l|l|}
\hline \multicolumn{1}{|c|}{ Composantes de l'anxiété } & \multicolumn{1}{c|}{ Items } \\
\hline la peur d'entrer en communication & $1,4,9,14,15,18,24,27,29,30,32$ \\
\hline la peur face aux tests & $3,5,6,8,10,11,12,16,17,20,21,22,25,26,28$ \\
\hline la peur d'être évalué négativement & $2,7,13,19,23,31,33$ \\
\hline
\end{tabular}


À chaque item du questionnaire, le sujet interrogé pouvait attribuer de 1 point à 5 points (au total il peut donc obtenir de 33 à 165 points). Dans la version originale du «FLCAS » un résultat inférieur correspondait à un niveau plus élevé d'anxiété langagière, par contre dans son adaptation française le nombre de points élevé signifie un haut degré d'anxiété. Pour calculer les résultats du questionnaire on a suivi la même procédure que J. E. Wilkinson (2011) : la réponse « Tout-à-fait d'accord » valait 5 points, et celle de « Tout-à-fait en désaccord » rapportait 1 point, à l'exception des items mentionnés négativement (par exemple "Commettre des erreurs en classe ne m'inquiète pas ") ou ayant une connotation négative par rapport à l'idée conductrice du questionnaire (par exemple « Normalement je suis à l'aise quand j'ai un contrôle en classe »). Ces items ont été évalués selon une pondération inverse : de 1 point pour la réponse «Toutà-fait d'accord » à 5 points pour celle de « Tout-à-fait en désaccord ». De plus, pour éviter tout malentendu et assurer une bonne compréhension du « FLCAS », ce questionnaire a été analysé du point de vue lexical avec chaque groupe interrogé avant de lui être remis. Les étudiants ont reçu une liste d'expressions tirées du questionnaire, et afin de leur fournir une explication voire une traduction adéquate, on leur a proposé de se référer à la page du Centre Nationale de Ressources Textuelles et Lexicales (www.cnrtl.fr). Cette phase a duré entre 15 et 20 minutes dans chaque groupe.

\section{ANALYSE DES RÉSULTATS}

\subsection{Les degrés d'anxiété langagière générale}

Pour mesurer les degrés d'anxiété langagière ressentie par les étudiants de la $1^{\text {ère }}$, de la $2^{\text {ème }}$ et de la $3^{\text {ème }}$ année d'études, il convenait de commencer par distinguer des degrés d'anxiété langagière : bas, moyen, haut. Le tableau (Tableau 2.) résume les résultats obtenus.

Tableau 2. La différenciation des degrés de l'anxiété langagière générale

\begin{tabular}{|c|c|c|c|}
\hline Degré & $\mathbf{1}^{\text {ère }}$ année & $\mathbf{2}^{\text {ème }}$ année & $\mathbf{3}^{\text {ème }}$ année \\
\hline Bas & $\begin{array}{c}\text { Tout score au dessous } \\
\text { de } 82 \text { pts }\end{array}$ & $\begin{array}{c}\text { Tout score au dessous } \\
\text { de } 78 \text { pts }\end{array}$ & $\begin{array}{c}\text { Tout score au dessous } \\
\text { de } 89 \text { pts }\end{array}$ \\
\hline Haut & $\begin{array}{c}\text { Tout score au dessus } \\
\text { de } 122 \text { pts }\end{array}$ & $\begin{array}{c}\text { Tout score au dessus } \\
\text { de } 114 \text { pts }\end{array}$ & $\begin{array}{c}\text { Tout score au dessus } \\
\text { de } 123 \text { pts }\end{array}$ \\
\hline Moyen & $\begin{array}{c}\text { Tout score } \\
\text { entre } 82 \text { et } 122 \text { pts }\end{array}$ & $\begin{array}{c}\text { Tout score } \\
\text { entre78 et } 114 \text { pts }\end{array}$ & $\begin{array}{c}\text { Tout score } \\
\text { entre } 89 \text { et } 123 \text { pts }\end{array}$ \\
\hline
\end{tabular}


Après avoir défini les catégories relatives à trois degrés d'anxiété langagière générale, il était possible d'y grouper les étudiants interrogés. Le diagramme ci-dessous présente les résultats obtenus, calculés en pourcentage (Diagramme 1.).

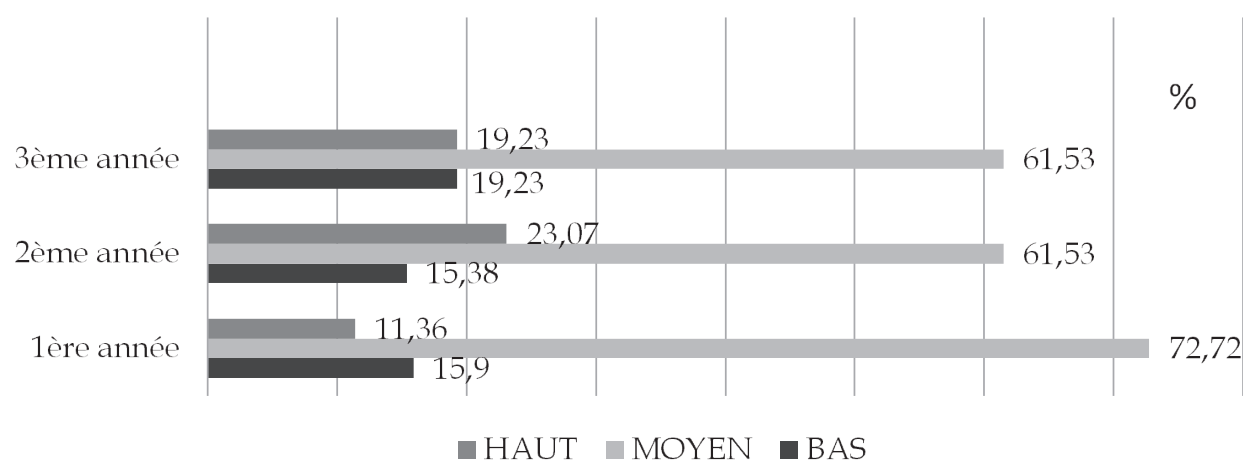

Diagramme 1. Les degrés d'anxiété langagière générale : $1^{\text {ère }}$ année, $2^{\text {ème }}$ année et $3^{\text {ème }}$ année

Ces résultats montrent bien un avantage certain du degré moyen d'anxiété langagière nuisible, quelle que soit l'année d'études. Pour la majorité et la grande majorité des étudiants interrogés, l'anxiété langagière générale correspond au degré moyen : en $1^{\text {ère }}$ année deux tiers des répondants $(72,72 \%)$ déclarent ressentir son degré comme moyen, en $2^{2 \text { eme }}$ et $3^{\text {zeme }}$ année ce pourcentage descend à $61,53 \%$. Le groupe intermédiaire en $1^{\text {ère }}$ année et le groupe avancé en $2^{\text {ème }}$ année sont presque unanimes dans leurs déclarations : 13 étudiants sur 14 en $1^{\text {ère }}$ année et 11 étudiants sur 14 en $2^{\text {ème }}$ année reconnaissent le degré d'anxiété langagière ressentie comme moyen. De plus, ce qui est intéressant à noter, c'est la fluctuation du pourcentage correspondant au haut degré d'anxiété langagière nuisible : il est le plus bas en $1^{\text {ère }}$ année et le plus élevé en $2^{\text {ème }}$ année, en $3^{\text {ème }}$ année il diminue légèrement pour atteindre $19,23 \%$. Les étudiants de la $1^{\text {ère }}$ année déclarant ressentir un bas degré d'anxiété langagière font tous partie du groupe débutant (cinq personnes).

Les résultats susmentionnés conduisent aux conclusions provisoires que chaque année d'études subséquente poserait aux étudiants interrogés de nouveaux défis considérables mettant à l'épreuve leurs compétences acquises, au point d'engendrer des peurs et inquiétudes relatives aux situations d'apprentissages particulières pour l'année donnée. Pour en savoir plus, passons à l'analyse des degrés de l'anxiété nuisible relative à la peur de communication, la peur face aux tests et la peur de l'évaluation négative. 


\subsection{La peur de communication, la peur face aux tests et la peur de l'évaluation négative}

Afin de comparer les degrés de la peur de communication, la peur face aux tests et la peur de l'évaluation négative en $1^{\text {ère }}, 2^{\text {ème }}$ et $3^{\text {ème }}$ année du cycle de licence, il était nécessaire d'établir des catégories relatives aux degrés bas, moyen et haut pour les composantes d'anxiété étudiées. ${ }^{2}$ On y a ensuite regroupé les étudiants des années interrogées (voir Tableau 3.).

Tableau 3. Les degrés de la peur de communication, de la peur face aux tests et de la peur de l'évaluation négative en $1^{\text {ère }}, 2^{2 \text { ème }}$ et $3^{\text {ème }}$ année d'études

\begin{tabular}{|c|c|c|c|c|c|c|c|}
\hline \multirow[t]{2}{*}{ Degré } & \multirow{2}{*}{$\begin{array}{l}\text { Composantes d'anxiété } \\
\text { langagière nuisible }\end{array}$} & \multicolumn{2}{|c|}{$\begin{array}{c}1^{\text {ère }} \text { année } \\
44 \mathrm{~N}\end{array}$} & \multicolumn{2}{|c|}{$\begin{array}{c}2^{\text {ème }} \text { année } \\
26 \mathrm{~N}\end{array}$} & \multicolumn{2}{|c|}{$\begin{array}{c}3^{3 \text { ème }} \text { année } \\
26 \mathrm{~N}\end{array}$} \\
\hline & & Nombre & $\%$ & Nombre & $\%$ & Nombre & $\%$ \\
\hline \multirow{3}{*}{ Haut } & peur d'entrer en communication & 5 & 11,36 & 1 & 3,85 & 4 & 15,38 \\
\hline & peur face aux tests & 5 & 11,36 & 4 & 15,38 & 3 & 11,53 \\
\hline & peur de l'évaluation négative & 5 & 11,36 & 5 & 19,23 & 2 & 7,69 \\
\hline \multirow{3}{*}{ Moyen } & peur d'entrer en communication & 34 & 77,27 & 20 & 76,92 & 19 & 73,07 \\
\hline & peur face aux tests & 33 & 75,00 & 18 & 69,23 & 20 & 76,92 \\
\hline & peur de l'évaluation négative & 31 & 70,45 & 19 & 73,06 & 18 & 69,23 \\
\hline \multirow{3}{*}{ Bas } & peur d'entrer en communication & 5 & 11,36 & 5 & 19,23 & 3 & 11,53 \\
\hline & peur face aux tests & 6 & 13,63 & 4 & 15,38 & 3 & 11,53 \\
\hline & peur de l'évaluation négative & 8 & 18,18 & 2 & 7,69 & 6 & 23,07 \\
\hline
\end{tabular}

Tout aussi bien que le degré d'anxiété langagière générale, le degré d'anxiété langagière relative à la peur de communication, la peur face aux tests et la peur de l'évaluation négative est ressenti comme étant moyen par la majorité ou la grande majorité des questionnés, quelle que soit l'année d'études. L'analyse détaillée révèle pourtant des fluctuations de résultats intéressantes, dressant un paysage de défis représentatifs pour les étudiants de chaque année d'études examinée.

Quant aux étudiants de $1^{\text {ère }}$ année, les cours de français dirigé représentent pour eux dans leur ensemble un défi important. Seulement cinq étudiants déclarent ressentir un bas degré d'anxiété relative aux situations de compréhension / expression orales, six le font pour les situations de tests et huit l'avouent pour les situations d'évaluation par l'enseignant et par les pairs. Ce dernier résultat indique quand même une acceptation légèrement plus élevée des situations d'évaluation en comparaison avec les situations de communication et de

\footnotetext{
${ }^{2}$ Pour les résultats des calculs détaillés voir Annexes 2 et 3.
} 
tests. Il est à noter que tous les questionnés déclarant ressentir un bas niveau d'anxiété relative aux types de situations d'apprentissage examinées font partie des groupes débutants. Contrairement aux intermédiaires et avancés qui ont plus à perdre s'il s'agit de l'image de leur compétences, les débutants peuvent toujours justifier leurs erreurs en se référant à leur manque de connaissance et d'expérience («je ne suis qu'un débutant»).

Ce qui semble un défi particulier pour les étudiants de $2^{\text {ème }}$ année, ce sont les situations de tests et celles d'évaluation par l'enseignant et par les pairs. Le premier type de situations constitue un défi pour 22 étudiants dont huit étudiants intermédiaires et 14 étudiants avancés. Tous les étudiants avancés et la moitié des intermédiaires déclarent ressentir un degré moyen d'anxiété nuisible relative à la peur face aux tests et les autres (quatre étudiants intermédiaires) identifient son degré comme haut. Le deuxième type de situations évoquées est un défi pour 24 questionnés dont 10 étudiants intermédiaires et 14 étudiants avancés. Le degré moyen de l'anxiété nuisible relative à la peur d'être évalué négativement est declaré par 11 étudiants avancés et huit étudiants intermédiaires. Le haut degré de la peur de l'évaluation négative est reconnu par deux étudiants du groupe intermédiaire et trois étudiants du groupe avancé. Vu ces résultats, il semble probable que la réussite à l'examen de 1 ère année engendre chez les étudiants interrogés la peur de perdre « leur status quo » de la réussite. Probablement, ils ne se sentent pas en sécurité et n'ont pas assez confiance en leurs compétences. Ils préféreraient donc éviter l'évaluation.

Quant à la $3^{\text {ème }}$ année, ce sont les situations de compréhension / expression orales et les situations de tests qui passent avant les situations d'évaluation par l'enseignant et par les pairs. L'un et l'autre type de situation d'apprentissage est reconnu comme une source importante d'anxiété nuisible par 23 étudiants. Le haut degré de la peur d'entrer en communication est déclaré par quatre d'entre eux et 19 avouent la ressentir au niveau moyen. Trois ressentent un haut degré de la peur face aux tests et 20 identifient son niveau comme moyen. Il est à préciser que les étudiants déclarant un haut niveau de l'anxiété nuisible relative aux situations de compréhension / expression orales et de tests ont tous commencé leur apprentissage du FLE en philologie romane. Comment expliquer ces résultats ?Pour les étudiants de $3^{\text {ème }}$ année, un défi considérable consiste à rédiger leur travail de licence. C'est la première fois qu'ils sont obligés de préparer un texte aussi long et exigeant du point de vue cognitif et langagier. Pendant les séminaires et les cours d'expression orale, leurs compétences de compréhension / expression orales sont donc mises à l'épreuve. Les maîtres de conférence leur demandent de comprendre des textes scientifiques, de respecter le système de conventions représentatives pour un discours scientifique et de prendre la parole 
pour faire une synthèse, rendre compte $d$ 'une lecture, donner leur avis, etc. Ceci paraît d'autant plus difficile pour les étudiants provenant des groupes débutants.

En somme, chaque année subséquante représente des défis nouveaux pour les étudiants interrogés, contrebalançant leur expérience d'études,leurs compétences acquises et suscitant de nouveau les peurs et les inquiétudes.

\section{CONCLUSION}

Les résultats de la présente étude confirment le clivage entre l'anxiété langagière observée chez certains étudiants en cycle de licence et les opportunités du développement offertes par les études en philologie romane. La familiarisation aux rituels universitaires, le développement de la performance langagière ainsi que les procédures et les techniques visant l'atténuation de l'anxiété langagière ne sont pas en mesure de niveler le degré d'anxiété langagière ressentie par les étudiants interrogés. Il semble que ces étudiants considèrent les situations d'apprentissage académique comme une menace pour leur bien-être, quelle que soit l'année interrogée. Par ailleurs, la mise en épreuve des savoirs et savoir-faire, qu'ils subissent chaque année d'études subséquante, s'avère grave au point de susciter leurs peurs, leurs inquiétudes et d'affecter leur joie d'apprentissage. Indépendamment de leur niveau, pour être contrôlées, les réactions émotionnelles dites négatives demandent de déléguer une partie des ressources, ce qui peut porter l'impact sur l'attention, la concentration et donner ainsi une fausse image de la performance des étudiants.

Cet état de fait confirme la réalité du défi pédagogique lié à la gestion de l'anxiété langagière nuisible par les étudiants interrogés en éveillant d'autres questions, à savoir :

1. Dans quelle mesure les étudiants interrogés se sont-ils familiarisés aux rituels universitaires?

2. Comment les perçoivent-ils (comme menaçants, amicaux ou neutres)?

3. Comment les étudiants évaluent-ils les procédures et techniques visant l'atténuation de l'anxiété langagière?

Les résultats du questionnaire au sujet de l'anxiété langagière nuisible ne fournissent pas des conclusions fiables et valides pour l'ensemble de la population étudiant le FLE dans le cadre universitaire. Ils encouragent pourtant à poursuivre les recherches. Il semble que le défi pédagogique n'est pas de proposer de nouveaux outils, techniques et façons de travailler canalisés sur la réduction d'anxiété langagière des étudiants mais de transformer leur approche de la tension et de l'appréhension ressenties en situations d'apprentissage : 
Ce que nous pensons de la tension ressentie affecte vraiment tout : du fonctionnement du système circulatoire au sens de la vie. La gestion la plus efficace du stress ne consiste pas tellement à le réduire ou à l'éviter mais à vérifier nos propres vues sur lui, et même à l'accepter ${ }^{3}$ (McGonigal 2016 : 22).

Pour reprendre la citation de S.S. Brevet, l'objectif général de la pédagogie orientée sur la gestion de l'anxiété langagière serait d'amener les étudiants à " se servir de leur anxiété pour intensifier leurs efforts en vue de la réussite » (Brever $2010: 77)$.

\section{RÉFÉRENCES}

Brewer, S. S. (2010). Un regard agentique sur l'anxiété langagière. In: J. Aden / T. Grimshaw / H. Penz / P. Lang (dir.). Enseigner les langues cultures à l'ère de la complexité: approches interdisciplinaires pour un monde en reliance. Teaching language and culture in an eraof complexity: Interdisciplinary approaches for an interrelated world (pp. 75-88). Brussels: Peter Lang.

Cadre européen commun de références pour les langues. Volume complémentaire avec de nouveaux descripteurs (2018). www.coe.int/cecr-volume-complementaire-avec-de-nouveaux-descripteurs / $16807875 \mathrm{~d} 5$ [access: 10.11.2019].

Daly, J. (1991). Understanding communication apprehension: An introduction for language educators. In: E. K. Horwitz / D. J. Young (dir.). Language anxiety: From theory and research to classroom implications, (pp. 3-14). Englewood CLIFFS, NJ: Prentice Hall.

Gardner, R. C. / MacIntyre, P. D. (1993). A student's contribution to second language acquisition, part II: Affective variables. Language Teaching, 26, 1-11. DOI: https://doi.org/10.1017/ S0261444800000045.

Hilleson, M. (1996). "I want to talk with them, but I don't want them to hear": An introspective study of second language anxiety in an English-medium school. In: K. Bailey / D. Nunan (dir.). Voices from the language classroom (pp. 248-275). Cambridge: Cambridge University Press.

Horwitz, E. K. / Horwitz, M. B. / Cope, J. A. (1986). Foreign language classroom anxiety. The Modern Language Journal, 70 (2), 125-132. DOI: https:// doi.org/10.2307/327317.

Jarie, L. / Bordás, C. S. / Orozco, A. M. / Supervía, P. U. (2019). Validación y Confiabilidad de la versión francesa de la escala de ansiedad lingüística Foreign Language Classroom Anxiety Scale y su aplicación a estudiantes franceses: revisión del análisis de Horwitz, Howitz y Cope, Aida y Pérez y Martínez Laurane Jarie, Carlos Salavera Bordás, Abel Merino Orozco y Pablo Usán Supervía. Thélème. Revista Complutense de Estudios Franceses, 34 (1), 207-225. DOI: http:/ / dx.doi.org/10.5209/THEL.61153.

Jebali, A. (2018). Anxiété langagière, communication médiée par les technologies et élicitation des clitiques objets du français L2. Alsic, 21/2018, mis en ligne le 20 novembre 2017, 1-19. http://journals.openedition.org/alsic/3164. DOI : https://doi.org/10.4000/alsic.3164 [access: 3.10.2019].

Kalińska-Łuszczyńska, S. (2015). L'acquisition d'une langue étrangère et l'anxiété langagière. Annales Universitatis Mariae Curie-Skłodowska.Sectio FF, Philologiae, 33, 35-47. DOI: https:/ / doi. org/ 10.17951/ff.2015.33.1.35.

\footnotetext{
${ }^{3}$ Traduction propre.
} 
Kalińska-Łuszczyńska, S. (2016). Les corrélats de l'anxiété langagière. Romanica Cracoviensia, 16 (2), 75-96. DOI: https:/ / doi.org/10.4467/20843917RC.16.008.5929.

Kolber, M. (2008). Zrozumieć lęk językowy. https:// repozytorium.ukw.edu.pl/handle/item/1805 [access: 1.09.2019].

Łuszczyńska, S. (2017). Impact de l'anxiété sur l'apprenatissage des langues. Studia Neofilologiczne, 13, 129-146. DOI: http:/ / dx.doi.org/10.16926/sn.2017.13.10.

McGonigal, K. (2016). Sita stresu. Jak stresować się mądrze i z pożytkiem dla siebie. Gliwice: Helion.

MacIntyre, P. D. (1995). How does anxiety affect second language learning? A reply to Sparks and Ganschow. The Modern Language Journal, 79 (1), 90-99. DOI: https://doi. org/10.1111/j.1540-4781.1995.tb05418.x.

MacIntyre, P. D. / Gardner, R. (1991). Methods and results in the study of anxiety in language learning: A review of the literature. Language Learning, 41, 85-117. DOI: https:/ doi. org/10.1111/j.1467-1770.1991.tb00677.x.

MacIntyre, P. D. / Gardner, R. C. (1994a). The effects of induced anxiety on cognitive processing in computerised vocabulary learning. Studies in Second Language Acquisition, 16, 1-17. DOI: https:/ / doi.org/10.1017/S0272263100012560.

MacIntyre, P. D. / Gardner R. C. (1994b). The subtle effects of language anxiety on cognitive processing in the second language. Language Learn, 44 (2), 283-305. DOI: https://doi. org/10.1111/j.1467-1770.1994.tb01103.x.

Matsuda, S. / Gobel, P. (2004). Anxiety and predictors of performance in the foreign language classroom. System, 32, 21-36. DOI: 10.1016/j.system.2003.08.002.

Phillips, E. M. (1991). Anxiety and oral competence: Classroom dilemma. French Review, 65, 1-14.

Porcher, L. (1995). Le français langue étrangère. Paris: Hachette Éducation.

Sarason, I. G. (1987). Test anxiety, cognitive interference, and performance. In: R. E. Snow / M. J. Farr (dir.). Aptitude, learning and instruction: Cognitive and affective process analyses, 3 (pp. 131-142). Hillsdale, N.J.: Erlbaum.

Scovel, T. (1978). The effect of affect: A review of the anxiety literature. Language Learning, 28, 129-142. DOI: https:/ / doi.org/10.1111/j.1467-1770.1978.tb00309.x.

Wilkinson, J. K. (2011). L'anxiété langagière chez les locuteurs d'anglais de niveau universitaire selon le programme d'apprentissage du français langue seconde préalablement suivi. Mémoire de Master de la Faculté des études supérieurs de l'Université Laval Québec, Linguistique et traduction. https:/ / corpus.ulaval.ca > jspui > bitstream [access: 15.10.2019].

Williams, K. (1991). Anxiety and formal second/foreign language learning. RELC Journal, 22 (2), 19-28. DOI: https:/ / doi.org/10.1177/003368829102200202.

Wine, J. D. (1971). Test anxiety and the direction of attention. Psychological Bulletin, 76, 992-104. DOI: https://dx.doi.org/10.1037/h0031332.

Young, D. J. (1986). The relationship between anxiety and foreign language oral proficiency ratings. Foreign Language Annals, 19 (5), 439-445. DOI: https:/ / doi.org/10.1111/j.1944-9720.1986. tb01032.x.

Young, D. J. (1992). Language anxiety from the foreign language specialist's perspective: Interviews with Krashen, Omaggio Hadley, Terrell, and Rardin. Foreign Language Annals, 25 (2), 157-172. DOI: https://doi.org/10.1111/j.1944-9720.1992.tb00524.x.

Zhang, L. J. (2001). Exploring variability in language anxiety: Two groups of PRC students learning ESL in Singapore. RELC Journal, 32 (1), 73-94. DOI: http:/ / dx.doi.org/10.1177/003368820103 200105. 


\begin{abstract}
ANNEXES
Annexe 1

À l'aide du questionnaire ci-dessous, évaluez votre degré d'accord ou de désaccord avec chacune des affirmations se référant à diverses situations de l'apprentissage d'une langue étrangère. Pour ce faire, à côté de chaque énoncé mettez la lettre correspondant au degré de votre accord ou votre désaccord : A - « Tout-à-fait d'accord »

$\mathrm{B}-\ll$ D'accord »

C - «Sans avis»

$\mathrm{D}$ - «En désaccord»

E - « Tout-à-fait en désaccord»
\end{abstract}

1. Je ne suis jamais complètement sûr de moi quand je parle une langue étrangère en classe.

2. Commettre des erreurs en classe ne m'inquiète pas.

3. Je tremble lorsque je sais que l'on va m'interroger en classe.

4. J'ai peur de ne pas comprendre ce que le professeur dit en $\mathrm{c}$ de langue étrangère.

5. Ça ne me dérangerait pas du tout d'avoir plus d'heures de cours de langue étrangère.

6. Pendant le cours, je me rends compte que je pense à d'autres choses qui n'ont rien à voir avec la matière.

7. Je pense que mes camarades de classe sont meilleurs que moi en langue étrangère.

8. Normalement je suis à l'aise quand j'ai un contrôle en classe.

9. Je deviens très nerveux quand je dois parler en classe et que je n'ai pas bien révisé.

10. Je crains les conséquences que peut avoir le fait de ne pas avoir la moyenne à une évaluation en langue étrangère.

11. Je ne comprends pas pourquoi certaines personnes se sentent aussi mal à cause des cours de langues étrangères.

12. En cours, je suis tellement nerveux que j'oublie certaines choses alors que je les connais.

13. Ça me gêne d'être volontaire en classe.

14. Je crois que j'aurais le trac si je devais parler en langue étrangère avec une personne native.

15. Ne pas comprendre ce que le professeur est en train de corriger me contrarie.

16. Je me contrarie en classe même si j'ai bien révisé le cours.

17. Très souvent, je n'ai pas envie d'aller en cours de langue étrangère.

18. Je suis sûr de moi au moment de prendre la parole en classe de langue étrangère.

19. Au moment de prendre la parole, j'ai peur que mon professeur me corrige chacune des fautes que je commettrai.

20. Mon cœur bat très fort quand je sais que l'on va me demander $\mathrm{d}^{\prime}$ intervenir en classe.

21. Plus j'étudie, plus je m'embrouille.

22. Je n'ai aucune pression ni aucune inquiétude au moment de réviser mes cours.

23. J'ai l'impression que mes camarades de classe parlent la langue étrangère mieux que moi.

24. Je suis très gêné quand je dois parler en langue étrangère devant les camarades.

25 . Les cours avancent tellement vite que j'ai peur de ne pas suivre le rythme.

26. En comparaison, je suis beaucoup plus nerveux en classe de langue étrangère que dans les autres cours ou dans mon travail personnel.

27. Je m'angoisse lorsque je parle en classe.

28. Avant d'entrer en classe, je me sens sûr de moi et détendu.

29. Je deviens nerveux lorsque je ne comprends pas chacun des mots que mon professeur dit.

30. La quantité de leçons que je dois apprendre pour pouvoir parler une langue étrangère me pèse. 
31. J'ai peur que mes camarades de classe se moquent de moi quand je parle en langue étrangère.

32. Je crois que je serai à l'aise en parlant avec des personnes natives qui utilisent la langue que je suis en train d'étudier.

33. Je me contrarie quand le professeur m'interroge sur des leçons que je n'ai pas pu réviser.

(Jarie et al. 2019)

\section{Annexe 2}

Tableau 4. Moyenne générale et écart type pour les composantes d'anxiété langagière nuisible

\begin{tabular}{|c|c|c|c|c|c|c|}
\hline \multirow{2}{*}{$\begin{array}{c}\text { Composantes d'anxiété langagière } \\
\text { nuisible }\end{array}$} & \multicolumn{3}{|c|}{ Moyenne générale } & \multicolumn{3}{|c|}{ Écart type } \\
\hline & $\begin{array}{c}1^{\text {ère }} \\
\text { année }\end{array}$ & $\begin{array}{c}2^{\text {ème }} \\
\text { année }\end{array}$ & $\begin{array}{c}3^{\text {ème }} \\
\text { année }\end{array}$ & $\begin{array}{c}1^{\text {ère }} \\
\text { année }\end{array}$ & $\begin{array}{c}2^{\text {ème }} \\
\text { année }\end{array}$ & $\begin{array}{c}3^{\text {ème }} \\
\text { année }\end{array}$ \\
\hline La peur d'entrer en communication & 35,11 & 31,88 & 36,3 & 6,67 & 8,38 & 6,42 \\
\hline La peur face aux tests & 44,79 & 42,53 & 46,34 & 9,06 & 10,05 & 7,49 \\
\hline La peur de l'évaluation négative & 22,09 & 19,57 & 20,96 & 5,80 & 5,43 & 4,57 \\
\hline
\end{tabular}

\section{Annexe 3}

Tableau 5. Les degrés de la peur de communication, de la peur face aux tests et la peur de l'évaluation négative

\begin{tabular}{|c|c|c|c|}
\hline \multirow{2}{*}{ Degré } & \multicolumn{3}{|c|}{ La peur d'entrer en communication } \\
\hline & $1^{\text {ère }}$ année & $2^{\text {ème }}$ année & $3^{\text {¿ème }}$ année \\
\hline Bas & Tout score au dessous de 28 & Tout score au dessous de 24 & Tout score au dessous de 30 \\
\hline Haut & Tout score au dessus de 42 & Tout score au dessus de 40 & Tout score au dessus de 43 \\
\hline Moyen & Tout score entre 28 et 42 & Tout score entre 24 et 40 & Tout score entre 30 et 43 \\
\hline \multirow{2}{*}{ Degré } & \multicolumn{3}{|c|}{ La peur face aux tests } \\
\hline & $1^{\text {ère }}$ année & $2^{\text {ème }}$ année & $3^{\text {ème }}$ année \\
\hline Bas & Tout score au dessous de 36 & Tout score au dessous de 32 & Tout score au dessous de 38 \\
\hline Haut & Tout score au dessus de 54 & Tout score au dessus de 53 & Tout score au dessus de 54 \\
\hline Moyen & Tout score entre 36 et 54 & Tout score entre 32 et 53 & Tout score entre 39 et 54 \\
\hline \multirow{2}{*}{ Degré } & \multicolumn{3}{|c|}{ La peur de l'évaluation negative } \\
\hline & $1^{\text {ère }}$ année & $2^{\text {ème }}$ année & $3^{\text {ème }}$ année \\
\hline Bas & Tout score au dessous de 16 & Tout score au dessous de 14 & Tout score au dessous de 16 \\
\hline Haut & Tout score au dessus de 28 & Tout score au dessus de 25 & Tout score 26 (arrondi à 26) \\
\hline Moyen & Tout score entre 16 et 28 & Tout score entre 14 et 25 & Tout score entre 16 et 26 \\
\hline
\end{tabular}

Received: 22.09.2019; revised: 21.03.2020

\section{MARZENA BLACHOWSKA-SZMIGIEL}

Uniwersytet im. Adama Mickiewicza w Poznaniu

majek@amu.edu.pl

ORCID: 0000-0001-6913-9711 
